\title{
10. THE EFFECT OF PITCH HEIGHT, TIMBRE AND OCTAVE ERROR ON ABSOLUTE PITCH ACCURACY. EDUCATIONAL IMPLICATIONS
}

\author{
Dorina Geta Iușcă ${ }^{317}$
}

\begin{abstract}
The accuracy of absolute pitch has often fallen into mythical perspectives, as this rare ability tends to fascinate people through its spectacular results. Many people tend to think that a musician with absolute pitch is always capable of identifying the musical note of any sound in any circumstances. The research literature has revealed that this is rarely true. Although there is a significant difference between real absolute pitch owners and pseudoabsolute pitch owners, the accuracy of absolute pitch is highly influenced by a series of musical factors such as: pitch chroma, pitch height and musical timbre. Therefore, it has been proven that the best absolute pitch accuracy manifests for medium pitch sounds, while very high or very low sounds tend to often be misidentified. Even more, absolute pitch owners tend to make an unusual mistake of misidentifying the octave. The familiar sounds (for example from the instrument the musician has studied in childhood) tend to produce less identification errors. Nevertheless, the piano timbre is usually associated with the best accuracy of absolute pitch. The aim of the present research is to synthetize up-to-date literature regarding the way these factors influence the accuracy of absolute pitch. The study focuses on the idea of normalizing the general perspective of absolute pitch accuracy, as musicians and teachers often tend to have very high expectations regarding this ability. The educational implications of the new perspective drawn here contribute to a better relation between teachers and students, as well as to a better understanding of this interesting musical ability.
\end{abstract}

Key words: absolute pitch accuracy, pitch height, pitch chroma, octave error, timbre

\section{Introduction}

The ability to identify sounds usually manifests itself in complex musical situations. Researchers have discovered that absolute pitch performance often depends on a series of factors related not only to the individual, but also to the musical context the person identifies the sounds into. These factors may influence absolute pitch accuracy so much that it even leads to a flexible way of defining this ability. Therefore, some researchers (Athos et al, 2007) choose to include absolute pitch owners on a continuous line rather than in two separate categories (musicians with absolute pitch versus musicians without absolute pitch).

Defining absolute pitch in this manner has repercussions on the way it is measured. Yes, there a very small number of musicians able to identify any sound in any type of situations. But in the same time, we cannot ignore the vast majority of absolute pitch owners whose accuracy depend on either pitch height,

317 Lecturer PhD., “George Enescu” National University of Arts from Iaşi, Romania, email: dorinaiusca@yahoo.com 
chroma or timbre. The present study focuses on creating a synthetic view on these musical factors that influence absolute pitch accuracy.

\section{The impact of pitch height on absolute pitch accuracy}

The human mind can detect and identify a vast range of sounds that stretches from $16 \mathrm{~Hz}$ (the lowest sound produced by the large pipe organ) up to $20.000 \mathrm{~Hz}$ (the sound of the fluorescent lights, the mosquito repellent device, the sounds produced by the pronunciation of $s, t, v$, the background sound of the TV). People are mostly sensitive to frequencies stretching from $220 \mathrm{~Hz}$ to 2048 $\mathrm{Hz}$, sounds that usually correspond to the human speaking voice. The instruments of the symphonic orchestra produce a large range of sounds. The lowest sound produced by the double-bass is $41 \mathrm{~Hz}$. The highest sound of the piccolo is $3951 \mathrm{~Hz}$. The highest musical sounds produced by a soprano usually situate around $1000 \mathrm{~Hz}$. The lowest sounds produced by a male voice situate around $70 \mathrm{~Hz}$ (ZyTrax, 2019) ${ }^{318}$. The cognitive perception of the sounds situated at the edge of the hearing spectrum is weak because people have trouble comparing their height (Urmă, 1982). Therefore, they are rarely used in music.

In order to identify two sounds as being different in height, they must be at a minimum frequency distance also named as differential pitch sensitivity that usually depends on register. For example, when comparing a sound of $1000 \mathrm{~Hz}$ to the closest higher one, the latter must be of $1003 \mathrm{~Hz}$. Also, the closest lower sound the we perceive as being different is of $997 \mathrm{~Hz}$. So, in the high musical range, a frequency no smaller than $3 \mathrm{~Hz}$ determines us to perceive two sounds as being different (Urmă, 1982).

Inside the musical range going from $500 \mathrm{~Hz}$ to $3000 \mathrm{~Hz}$, the human ear can detect about 232 different sounds. In this musical range, musicians may be capable to detect 20 sounds inside a semitone. But this is not true for a lower range of sounds. For example, in the lowest piano octave (that stretches from $32,70 \mathrm{~Hz}$ up to $61.74 \mathrm{~Hz}$ ) musicians can detect only 58 sounds (Urmă, 1982).

When referring to the whole audible spectrum, an ordinary musician can differentiate around 1450 sounds from which only 109 are usually used in academic music. Therefore, absolute pitch owners tend to memorize around 100 musical sounds.

The highest accuracy of absolute pitch situates in the medium range sounds (Takeuchi \& Hulse, 1993). Moreover, in this range, musicians with absolute pitch have the highest reaction speed when identifying the sounds (idem). In the same time, we must consider that every musical instrument has its own range and the experience with it creates a better accuracy of absolute pitch for its specific range. Therefore, Takeuchi and Hulse (1993) have discovered two relevant cases: one of double bass player whose absolute pitch accuracy was the best for the low range sounds, and another case of a violin player whose

318 http://www.zytrax.com/tech/audio/audio.html 
absolute pitch accuracy was for the high range sounds and not for the medium range sounds.

In the case of very high or very low sounds, absolute pitch accuracy significantly drops. Two French researchers (Semal \& Demany, 1990) have discovered that sounds higher than $4000 \mathrm{~Hz}$ (interestingly, the highest piano sound is of $4186 \mathrm{~Hz}$ ) cannot be identified by musicians with absolute pitch and tend to correspond to the same musical note even when they are different in height. This psychological phenomenon may be explained through the fact that the differential pitch sensitivity is much lower for these extreme sounds, especially in the case of older musicians (Ross et al, 2004; Athos et al, 2007; Takeuchi \& Hulse, 1993). This is why absolute pitch musicians older than 45 tend to name the sound one or two semitones higher (Parncutt \& Levitin, 1999; Takeuchi \& Hulse, 1993; Baharloo et al, 1998; Athos et al, 2007).

A study (Athos et al, 2007) conducted at the University of California on 981 absolute pitch musicians has discovered that subjects around 45-50 years old tended to locate the musical notes one semitone higher than the real notes. The subject around 60-70 years old misidentified the notes two semitones higher. This error has been made on piano and pure (electronic sounds with no harmonics) sounds.

\section{The influence of timbre on absolute pitch accuracy}

The timbre of a musical sound is often described in terms of a sum of several distinct frequencies called harmonics that accompany the fundamental frequency (Giuleanu, 1975). Although there are some musicians capable of identifying any type of sounds, researchers (Takeuchi \& Hulse, 1993; Miyazaki \& Ogawa, 2006; Lockhead \& Byrd, 1981; Parncutt \& Levitin, 1999; Athos et al, 2007; Zatorre, 2003; Vanzella \& Schellenberg, 2010) have found that absolute pitch owners tend to be influenced by timbre when naming the musical notes. For example, an American study (Hsieh \& Saberi, 2009) has utilized 50 piano sounds and 50 pure sounds in order to test the accuracy of absolute pitch owners and obtained performance of more than $80 \%$. In the second phase of the study, researchers modified the experimental sounds by taking out the fundamental frequency and some harmonics. The results have shown that absolute pitch accuracy significantly dropped down to $60 \%$ as the number of harmonics decreased. The study has shown that timbre may offer enough information to absolute pitch musicians in order to affect pitch identification.

Several researchers (Miyazaki \& Ogawa, 2006; Lockhead \& Byrd, 1981; Parncutt \& Levitin, 1999; Takeuchi \& Hulse, 1993) have noticed that absolute pitch accuracy is higher for the sounds of the instrument musicians studied. The connection of pitch and timbre is therefore once again revealed and it may be neuro-psychologically explained (Menon et al, 2002; Zatorre \& Belin, 2001) through similar processing mechanisms. In 1951, Brammer (apud Takeuchi \& Hulse, 1993) have discovered on a group of 42 absolute pitch violinists that they 
identified violin sounds significantly better than clarinet sounds. The effect of timbre familiarity may go even further. Sergeant (1969 apud Takeuchi \& Hulse, 1993) indicated the case of an absolute pitch pianist who identified the sounds of her own piano significantly better than the sounds of other pianos.

A group of scientists from Berlin (Schlemmer, Kulke, Kuchinke \& Van der Meer, 2005) has used 88 musical sounds from symphonic orchestra instruments presented for 2 seconds to 21 absolute pitch musicians and measured pupillary response in order to evaluate the level of mental effort. Previous studies (Granholm \& Steinhauer, 2004 apud Schlemmer et al, 2005) had indicated that higher levels of cognitive effort significantly correlate with higher pupillary response. Results have shown that subject identified the familiar sounds of the instrument they studied with more accuracy, smaller reaction time and lower pupillary response. Patricia Vanzella and Glen Schellenberg (2010) have on-line tested 198 absolute pitch owners and discovered that the accuracy of absolute pitch may also depend on the first instrument they studied in childhood. Accordingly, the subjects who studied piano during childhood have revealed higher absolute pitch accuracy than the subjects who studied other instruments.

Many sources (Takeuchi and Hulse, 1993; Vanzella \& Schellenberg, 2010; Schlemmer et al, 2005; Hsieh \& Saberi, 2009) have found that piano sounds are the easiest to identify. Miyazaki and Ogawa (2006) explain this result through the fact that the piano is the most utilized musical instrument due to its large versatility in music education. Dixon Ward (1963 apud Miyazaki \& Ogawa, 2006) even created the name "absolute piano" in order to describe a certain category of absolute pitch musicians with greater accuracy for piano notes and weaker accuracy for other instrumental sounds. Interestingly, most experimental studies used piano sounds when measured absolute pitch, probably due to their accessibility. This is why some researchers (Vanzella \& Schellenberg, 2010) sustain that these studies measured "absolute piano" and not absolute pitch.

Not all absolute pitch musicians are affected by timbre. Athos et al (2007) found that $44 \%$ of them are capable of equally identify any type of sounds. Also, Baharloo et al (1998), from 612 questioned absolute pitch musicians, 79 procent of them have declared equal accuracy for all kinds of instrumental sounds. Other experiments (Miyazaki, 1989; Miyazaki \& Oagwa, 2006; Athos et al, 2007; Lockhead \& Byrd, 1981) have compared acoustic piano sounds with electronic piano sounds and found better accuracy of absolute pitch for acoustic piano sounds. Nevertheless, in comparison to piano sounds, vocal sounds are significantly harder to name (Athos et al, 2007; Vanzella \& Schellenberg, 2010). The instability of the fundamental sound and the vocal vibrato create perceptual confusion to absolute pitch owners.

\section{Octave error in absolute pitch musicians}

The sounds of audible human spectrum $(16 \mathrm{~Hz}-20.000 \mathrm{~Hz})$ are spread across 11 octaves. The highest two octaves ( $8000 \mathrm{~Hz}$ and above) are rarely used 
in academic music, especially as these sounds can only be produce by electronic instruments. The remaining nine octaves correspond to the range of the large pipe organ and are covered by different symphonic instruments.

When measuring absolute pitch, most experiments (Miyazachi \& Ogawa, 2006; Vanzella \& Schellenberg, 2010; Benguerel \& Westadal, 1991; Takeuchi \& Hulse, 1991; Takeuchi \& Hulse, 1993; Bermudez \& Zatorre, 2009; Schlemmer et al, 2005) used sounds from three medium octaves, situated from $100 \mathrm{~Hz}$ up to $1000 \mathrm{~Hz}$. Still, there are some studies (Lockhead \& Byrd, 1981; Miyazachi, 1989) that used 5 medium octaves with sounds from $50 \mathrm{~Hz}$ up to $2000 \mathrm{~Hz}$.

One of the curiosities noticed by researchers is that absolute pitch musicians tend to misidentify the octave of the sound, although they name the pitch chroma perfectly. This phenomenon has been named octave error. When asked to identify the octave of the perceived sounds, musicians with and without absolute pitch make the same mistakes (Lockhead \& Byrd, 1981; Takeuchi \& Hulse, 1993). The possible explanations of octave error refer to:

- the cognitive overload determined by two different mental processes (Miyazachi, 1989);

- absolute pitch musicians may confuse the fundamental sound with the first harmonic situated one octave higher (Ward \& Bunrs, 1982);

- in unfamiliar instruments sounds may appear higher due to the specific timbre (Athos et al, 2007);

- the existence of different octave naming systems in international studies may confuse absolute pitch musicians familiar to a certain octave naming system (Athos et al, 2007).

Due to this octave error, many researchers suggest that when measuring absolute pitch is it better not to ask for the pitch height, but only the pitch chroma, as identifying the octave where the sound is situated may constitute a different cognitive task not necessarily related to absolute pitch.

\section{Conclusions and educational implications}

Absolute pitch is a well desired ability among musicians due to its benefic effects in reading and writing music. Nevertheless, experimental studies have shown that absolute pitch musicians are not perfect in identifying sounds, as their accuracy depend either on pitch height, timbre and octave error. This is why experts in music and in education should consider absolute pitch a rare but not idealized ability. It is simple developed in some students and it should not be the purpose of music education. Relative pitch is always a good way to identify musical sounds, by using a reference note which is usually $l a$. The cognitive processes involved in relative pitch are more complex and it involves theoretical information at a higher level. 


\section{References}

1. Athos, A., Levinson, B., Kistler, A., Zemansky, J., Bostrom, A., Freimer, N., Gitschier, J. (2007). Dichotomy and perceptual distortions in absolute pitch ability. PNAS, 104(37), 14795-14800.

2. Baharloo, S., Johnston, P., Service, S., Gitschier, J., Freimer, N. (1998). Absolute pitch: An approach for identification of genetic and nongenetic components. American Journal of Human Genetics, 62, 224-231.

3. Giuleanu, V. (1975). Principii fundamentale în teoria muzicii, București: Muzicală.

4. Granholm, E. \& Steinhauer, S.R. (2004). Introduction: Pupillometric measures of cognitive and emotional processing. International Journal of Psychophysiology, 52, 1-6.

5. Hsieh, I. \& Saberi, K. (2009). Virtual pitch extraction from harmonic structures by absolute-pitch musicians. Acoustical Physics, 55(2), 232-239.

6. Lockhead, G. \& Byrd, R. (1981). Practically perfect pitch. Journal of Acoustical Society of America, 70(2), 387-389.

7. Menon, V., Levitin, D., Smith, B.K., Lembke, A., Krasnov, B.D., Glazer, D., Glover, G.H., McAdams, S. (2002). Neural correlates of timbre change in harmonic sounds. NeuroImage, 17, 1742-1754.

8. Miyazachi, K. \& Ogawa, Y. (2006). Learning absolute pitch by children: A cross-sectional study. Music Perception, 24(1), 63-78.

9. Miyazachi, K. (1989). Absolute pitch identification: Effects of timbre and pitch region. Music Perception, 7(1), 1-14.

10. Parncutt, R. \& Levitin, D. (1999). Absolute pitch. Grove Dictionary.

11. Schlemmer, K., Kulke, F., Kuchinke, L., Meer, E. (2005). Absolute pitch and pupillary response: Effects of timbre and key color. Psychophysiology, 42, 465-472.

12. Semal, C. \& Demany, L. (1990). The upper limit of "musical" pitch. Music Perception, 8(2), 165-176.

13. Takeuchi, A. \& Hulse, S. (1993). Absolute pitch. Psychological Bulletin, 113(2), 345-361.

14. Urmă, D. (1982). Acustică și muzică. București: Științifică și enciclopedică.

15. Vanzella, P. \& Schellenberg E. (2010). Absolute pitch: Effects of timbre on note-naming ability. PLoS ONE, 5(11), e15449.

16. Zatorre, R. (2003). Absolute pitch: A model for understanding the influence of genes and development on neural and cognitive function. Nature Neuroscience, 6(7), 692-695.

17. Zattore, R.J., Belin, P. (2001). Spectral and temporal processing in human auditory cortex. Cerebral Cortex, 11, 946-953. 